\title{
OGLE-2014-BLG-0289: Precise Characterization of a Quintuple-peak Gravitational Microlensing Event
}

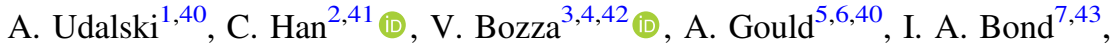 \\ and
}

P. Mróz ${ }^{1}$, J. Skowron ${ }^{1}$ (D), Ł. Wyrzykowski ${ }^{1}$, M. K. Szymański ${ }^{1}$, I. Soszyński ${ }^{1}$, K. Ulaczyk ${ }^{1}$, R. Poleski $^{1,6}$, P. Pietrukowicz $^{1}{ }^{\text {(D) }}$, S. Kozłowski ${ }^{1}$

(The OGLE Collaboration),

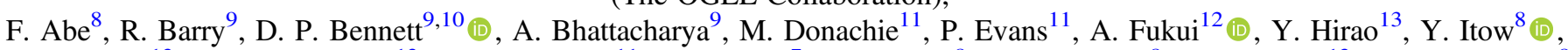
K. Kawasaki ${ }^{13}$ (D), N. Koshimoto ${ }^{13}$ (D), M. C. A. Li ${ }^{11}$, C. H. Ling ${ }^{7}$, K. Masuda ${ }^{8}$, Y. Matsubara ${ }^{8}$, S. Miyazaki ${ }^{13}$ (D), H. Munakata ${ }^{8}$, Y. Muraki ${ }^{8}$, M. Nagakane ${ }^{13}$, K. Ohnishi ${ }^{14}$, C. Ranc ${ }^{9}$, N. Rattenbury ${ }^{11}$, T. Saito ${ }^{15}$, A. Sharan ${ }^{11}$, D. J. Sullivan ${ }^{16}$, T. Sumi ${ }^{13}$, D. Suzuki ${ }^{17}$ (D), P. J. Tristram ${ }^{18}$, T. Yamada ${ }^{13}$, A. Yonehara ${ }^{19}$

(The MOA Collaboration),

R. A. Street $^{20}$, Y. Tsapras $^{21}$, E. Bachelet ${ }^{20}$ (D) D. M. Bramich ${ }^{22}$, G. DÁgo ${ }^{3,23}$, M. Dominik ${ }^{24}$, R. Figuera Jaimes ${ }^{24,25}$, K. Horne ${ }^{24}$, M. Hundertmark ${ }^{26,27}$, N. Kains ${ }^{28}$ (D), J. Menzies ${ }^{29}$, R. Schmidt $^{30}$, C. Snodgrass ${ }^{31,32}$ (iD) I. A. Steele ${ }^{33}$ (D), J. Wambsganss ${ }^{30}$ (iD

(Robonet Collaboration),

R. W. Pogge ${ }^{6}$ (iD, Y. K. Jung ${ }^{34}$, I.-G. Shin $^{34}$, J. C. Yee ${ }^{34}$, W.-T. Kim ${ }^{35}$

(The $\mu$ Fun Collaboration),

and

C. Beichman ${ }^{36}$, S. Carey ${ }^{37}$, S. Calchi Novati ${ }^{3,38}$, and W. Zhu ${ }^{6,39}$

(The Spitzer Team)

\author{
${ }^{1}$ Warsaw University Observatory, Al. Ujazdowskie 4, 00-478 Warszawa, Poland \\ ${ }^{2}$ Department of Physics, Chungbuk National University, Cheongju 28644, \\ Republic of Korea; cheongho@astroph.chungbuk.ac.kr \\ ${ }^{3}$ Dipartimento di Fisica "E. R. Caianiello," Universitá di Salerno, Via Giovanni Paolo II, \\ I-84084 Fisciano (SA), Italy \\ ${ }^{4}$ Istituto Nazionale di Fisica Nucleare, Sezione di Napoli, Via Cintia, I-80126 Napoli, Italy \\ ${ }^{5}$ Korea Astronomy and Space Science Institute, Daejon 34055, Republic of Korea \\ ${ }^{6}$ Department of Astronomy, Ohio State University, 140 West 18th Avenue, Columbus, OH 43210, USA \\ 7 Institute of Natural and Mathematical Sciences, Massey University, Auckland 0745, New Zealand \\ ${ }^{8}$ Institute for Space-Earth Environmental Research, Nagoya University, 464-8601 Nagoya, Japan \\ ${ }^{9}$ Code 667, NASA Goddard Space Flight Center, Greenbelt, MD 20771, USA \\ ${ }^{10}$ Department of Physics, University of Notre Dame, 225 Nieuwland Science Hall, Notre Dame, IN 46556, USA \\ ${ }_{11}$ Dept. of Physics, University of Auckland, Private Bag 92019, Auckland, New Zealand \\ ${ }^{12}$ Okayama Astrophysical Observatory, National Astronomical Observatory of Japan, Asakuchi, \\ 719-0232 Okayama, Japan \\ ${ }^{13}$ Department of Earth and Space Science, Graduate School of Science, Osaka University, Toyonaka, \\ Osaka 560-0043, Japan \\ ${ }^{14}$ Nagano National College of Technology, 381-8550 Nagano, Japan \\ ${ }^{15}$ Tokyo Metroplitan College of Industrial Technology, 116-8523 Tokyo, Japan \\ ${ }^{16}$ School of Chemical and Physical Sciences, Victoria University, Wellington, New Zealand \\ ${ }^{17}$ Institute of Space and Astronautical Science, Japan Aerospace Exploration Agency, Kanagawa 252-5210, Japan \\ ${ }^{8}$ Mt. John University Observatory, P.O. Box 56, Lake Tekapo 8770, New Zealand \\ ${ }^{19}$ Department of Physics, Faculty of Science, Kyoto Sangyo University, 603-8555 Kyoto, Japan \\ ${ }^{20}$ Las Cumbres Observatory Global Telescope Network, 6740 Cortona Drive, suite 102, Goleta, CA 93117, USA \\ ${ }^{21}$ Zentrum für Astronomie der Universität Heidelberg, Astronomisches Rechen-Institut, Mönchhofstr. 12-14, 69120 Heidelberg, Germany \\ ${ }^{22}$ New York University Abu Dhabi, Saadiyat Island, Abu Dhabi, P.O. Box 129188, United Arab Emirates \\ ${ }^{23}$ Istituto Nazionale di Fisica Nucleare, Sezione di Napoli, Napoli, Italy \\ ${ }^{24}$ School of Physics \& Astronomy, University of St. Andrews, North Haugh, St. Andrews KY 16 9SS, UK \\ ${ }^{25}$ European Southern Observatory, Karl-Schwarzschild-Str. 2, D-85748 Garching bei Munchen, Germany \\ ${ }^{26}$ Niels Bohr Institute, University of Copenhagen, Juliane Maries Vej 30, DK-2100, Kobenhavn, Denmark \\ ${ }^{27}$ SUPA, School of Physics \& Astronomy, University of St. Andrews, North Haugh, St. Andrews KY16 9SS, UK \\ ${ }^{28}$ Space Telescope Institute, 3700 San Martin Drive, Baltimore, MD 21218, USA \\ ${ }^{29}$ South African Astronomical Observatory, P.O. Box 9, Observatory 7935, South Africa \\ ${ }^{30}$ Astronomisches Rechen-Institut, Zentrum für Astronomie der Universit at Heidelberg (ZAH), \\ D-69120 Heidelberg, Germany \\ ${ }^{31}$ Planetary and Space Sciences, Dept of Physical Sciences, The Open University, Milton Keynes, \\ MK7 6AA, UK \\ ${ }^{32}$ Max Planck Institute for Solar System Research, Justus-von-Liebig-Weg 3, D-37077 Gotingen, Germany \\ ${ }^{33}$ Astrophysics Research Institute Liverpool John Moores University, Liverpool L3 5RF, UK \\ ${ }^{34}$ Harvard-Smithsonian Center for Astrophysics, 60 Garden Street, Cambridge, MA 02138, USA \\ ${ }^{35}$ Department of Physics \& Astronomy, Seoul National University, Seoul 151-742, Republic of Korea
}




\author{
${ }^{36}$ NASA Exoplanet Science Institute, California Institute of Technology, Pasadena, CA 91125, USA \\ ${ }^{37}$ Spitzer Science Center, MS 220-6, California Institute of Technology, Pasadena, CA 91125 USA \\ ${ }^{38}$ IPAC, Mail Code 100-22, California Institute of Technology, 1200 East California Boulevard, Pasadena, CA 91125, USA \\ ${ }^{39}$ Canadian Institute for Theoretical Astrophysics, 60 St. George Street, University of Toronto, Toronto, ON M5S 3H8, Canada \\ Received 2017 November 10; revised 2017 December 11; accepted 2017 December 11; published 2018 January 24
}

\begin{abstract}
We present the analysis of the binary-microlensing event OGLE-2014-BLG-0289. The event light curve exhibits five very unusual peaks, four of which were produced by caustic crossings and the other by a cusp approach. It is found that the quintuple-peak features of the light curve provide tight constraints on the source trajectory, enabling us to precisely and accurately measure the microlensing parallax $\pi_{\mathrm{E}}$. Furthermore, the three resolved caustics allow us to measure the angular Einstein radius $\theta_{\mathrm{E}}$. From the combination of $\pi_{\mathrm{E}}$ and $\theta_{\mathrm{E}}$, the physical lens parameters are uniquely determined. It is found that the lens is a binary composed of two $M$ dwarfs with masses $M_{1}=0.52 \pm 0.04 M_{\odot}$ and $M_{2}=0.42 \pm 0.03 M_{\odot}$ separated in projection by $a_{\perp}=6.4 \pm 0.5$ au. The lens is located in the disk with a distance of $D_{\mathrm{L}}=3.3 \pm 0.3 \mathrm{kpc}$. The reason for the absence of a lensing signal in the Spitzer data is that the time of observation corresponds to the flat region of the light curve.
\end{abstract}

Key words: binaries: general - gravitational lensing: micro

Supporting material: data behind figure

\section{Introduction}

Since commencing in the early 1990s (Udalski et al. 1994; Alcock et al. 1995; Aubourg et al. 1995), massive surveys have detected numerous microlensing events. The detection rate of microlensing events, which was of order $10 \mathrm{yr}^{-1}$ in the early stage of the surveys, has greatly increased and currently more than 2000 events are annually detected.

However, determinations of lens masses have been possible for very limited cases. The difficulty of the lens mass measurement arises because the event timescale, which is the only measurable quantity related to the lens mass for general lensing events, is related to not only the mass $M$ but also to the relative lens-source proper motion $\mu$ and the lenssource parallax $\pi_{\text {rel }}$, i.e.,

$$
t_{\mathrm{E}}=\frac{\sqrt{\kappa M \pi_{\text {rel }}}}{\mu} ; \quad \pi_{\text {rel }}=\mathrm{au}\left(\frac{1}{D_{\mathrm{L}}}-\frac{1}{D_{\mathrm{S}}}\right),
$$

where $\kappa=4 G /\left(c^{2} \mathrm{au}\right)$, and $D_{\mathrm{L}}$ and $D_{\mathrm{S}}$ represent the distances to the lens and source, respectively. For the unique determination of the lens mass, one needs to measure two additional observables of the angular Einstein radius $\theta_{\mathrm{E}}$ and the microlens parallax $\pi_{\mathrm{E}}$, i.e.,

$$
M=\frac{\theta_{\mathrm{E}}}{\kappa \pi_{\mathrm{E}}},
$$

where $\theta_{\mathrm{E}}^{2}=\kappa M \pi_{\text {rel }}$ (Gould 2000).

The angular Einstein radius can be measured by detecting light curve deviations caused by finite-source effects (Gould 1994; Nemiroff \& Wickramasinghe 1994). For lensing events produced by single masses, finite-source effects can be detected when a lens crosses the surface of a source star (Pratt et al. 1996; Choi et al. 2012). However, the ratio of the angular source radius $\theta_{*}$ to the angular Einstein radius is of order $10^{-3}$ for a main-sequence source star and of order $10^{-2}$ even for a giant star. Therefore, the chance to detect finite-source effects for a single-lens event is very low.

\footnotetext{
${ }^{40}$ The $\mu$ FUN Collaboration.

41 Corresponding author.

42 The RoboNet Collaboration.

43 The MOA Collaboration.
}

For events produced by binary objects, on the other hand, the probability of $\theta_{\mathrm{E}}$ measurement is relatively high because binary-lens events usually produce caustic-crossing features from which finite-source effects can be detected.

One can measure the microlens parallax from the light curve deviation induced by the acceleration of the source motion caused by the Earth's orbital motion: "annual microlens parallax" (Gould 1992). One can also measure the microlens parallax by simultaneously observing a lensing event from the ground and from a satellite in a heliocentric orbit: "space-based microlens parallax" (Refsdal 1966; Gould 1994). Considering that the physical Einstein radius $r_{\mathrm{E}}=D_{\mathrm{L}} \theta_{\mathrm{E}}$ of typical Galactic lensing events is of order a few $\mathrm{au}$, for satellites with a projected Earth-satellite separation of order au, the light curves seen from the Earth and the satellite usually exhibit considerable differences, e.g., OGLE-2015BLG-0124 (Udalski et al. 2015), OGLE-2015-BLG-0966 (Street et al. 2016), OGLE-2015-BLG-1268, and OGLE2015-BLG-0763 (Zhu et al. 2016), and this enables a precise measurement of $\pi_{\mathrm{E}}$. By contrast, deviations in lensing light curves induced by annual microlens-parallax effects are in most cases very subtle due to the small positional change of the Earth during $\sim(O)$ 10-day durations of typical lensing events. Furthermore, parallax-induced deviations can often be confused with deviations caused by other higher-order effects such as the orbital motion of the lens (Batista et al. 2011; Skowron et al. 2011; Han et al. 2016b). As a result, measurements of annual microlens parallaxes are in many cases subject to large uncertainty both in precision and accuracy.

It was pointed out by An \& Gould (2001) that the chance to determine the lens mass by measuring both $\pi_{\mathrm{E}}$ and $\theta_{\mathrm{E}}$ is high for a subclass of binary lensing events with three wellmeasured peaks where two peaks are produced by caustic crossings and the other by a cusp approach. This is because the individual peaks provide tight constraints on the source trajectory, enabling one to measure the microlens parallax. Furthermore, the angular Einstein radius is measurable from the analysis of almost any well-resolved caustic crossing, making triple-peak events good candidates for lens mass measurements. 

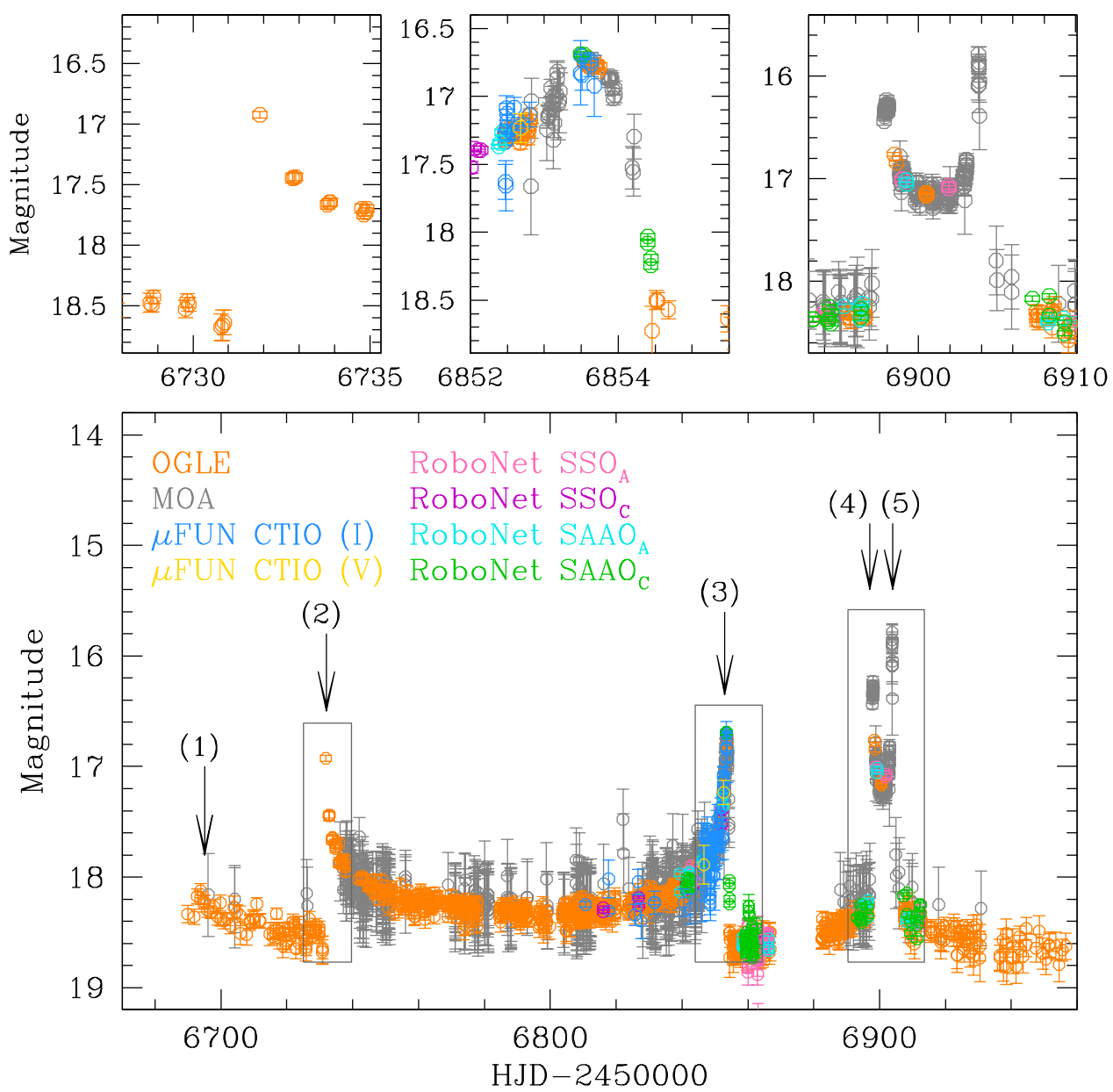

Figure 1. Light curve of OGLE-2014-BLG-0289. The upper panels show the zoom of the regions enclosed by boxes in the lower panel. The numbered arrows designate the peaks in the light curve. The numbered arrows indicate the locations of the five peaks. The data used to create this figure are available.

In this paper, we present the analysis of the binary-lens event OGLE-2014-BLG-0289. The light curve of the event exhibits five very unusual peaks. Among these peaks, four were produced by caustic crossings and the other by a cusp approach. The angular Einstein radius is precisely measured by detecting finite-source effects from the resolutions of three caustic crossings. Furthermore, the well-resolved multiple peaks enable us to measure the microlens parallax, leading to an accurate and precise measurement of the lens mass.

\section{Observation and Data}

The source star of the microlensing event OGLE-2014-BLG0289 is located toward the Galactic bulge field. The equatorial coordinates of the source star are (R.A., decl.) $)_{2000}=$ (17:53:51.66, -29:05:05.6), which correspond to Galactic coordinates $(l, b)=\left(0.80,-1^{\circ} .62\right)$. The magnification of the source flux caused by lensing was found on 2014 March 17 $\left(\mathrm{HJD}^{\prime}=\mathrm{HJD}-2450000 \sim 6733\right)$ by the Early Warning System of the Optical Gravitational Lensing Experiment (OGLE; Udalski 2003) survey from observations conducted using the $1.3 \mathrm{~m}$ Warsaw Telescope at the Las Campanas Observatory in Chile. The event was also in the footprint of the Microlensing Observations in Astrophysics (MOA; Bond et al. 2001; Sumi et al. 2003) survey that was conducted using the $1.8 \mathrm{~m}$ telescope at Mt. John Observatory in New Zealand.
The event was dubbed MOA-2014-BLG-092 in the "MOA Transient Alerts" list. ${ }^{44}$ Data from the OGLE and MOA surveys were acquired in the the standard Cousins $I$ and the customized MOA $R$ passband, respectively. Figure 1 shows the light curve of the event.

The source flux was already magnified before the 2014 Bulge season started. Just one day before the event was identified, i.e., HJD' $\sim 6732$, the light curve exhibited a sharp spike. Such a spike feature commonly appears in binary lensing events and is produced when a source crosses the caustic of a binary lens. Caustics indicate source positions at which the flux of a point source is infinitely magnified. Caustic crossings in binary-lens events occur in pairs because binary caustics form closed curves. When the source passes the inner region of a caustic, the light curve exhibits a characteristic U-shape trough followed by another spike that occurs when the source exits the caustic. The event followed this U-shape pattern of a binary lensing event until another spike appeared at $\mathrm{HJD}^{\prime} \sim 6854$.

The event was analyzed in real time with its progress. On 2014 June $18\left(\mathrm{HJD}^{\prime} \sim 6827\right)$, when the event passed the bottom of the U-shape trough, the first model was announced to the microlensing community by C. Han and V. Bozza. According to this model, the spike in the light curve was

\footnotetext{
${ }^{44}$ http://www.massey.ac.nz/iabond/moa/alerts/listevents.php?year=2014
} 
produced by the crossing of the source over the single large caustic formed by a lens that consisted of two similar masses separated in projection by $\sim \theta_{\mathrm{E}}$. Real-time modeling was important in preparing follow-up observations to resolve the caustic crossing, which yields the angular Einstein radius. It also helped to prepare space-based observations using the Spitzer telescope, which was separated $\sim 1$ au from the Earth at the time of the event. Caustic-crossing binary-lens events are important targets of Spitzer observations because one can measure the space-based microlens parallax, and the measured $\pi_{\mathrm{E}}$ combined with $\theta_{\mathrm{E}}$ leads to the measurement of the lens mass. Due to these considerations, real-time modeling was conducted more frequently as the source approached closer to the caustic exit.

The caustic exit occurred at $\mathrm{HJD}^{\prime} \sim 6854$, which matched approximately the time predicted by modeling. Follow-up observations were conducted by two groups including the Microlensing Follow-Up Network ( $\mu$ FUN) and RoboNet. The $\mu$ FUN group observed the event using the $1.3 \mathrm{~m}$ SMART telescope at the CTIO Observatory in Chile. The RoboNet observations were conducted with the $1 \mathrm{~m}$ robotic telescopes at South African Astronomical Observatory (SAAO) in South Africa and Siding Spring Observatory (SSO) in Australia. $\mu$ FUN observations were conducted in standard Cousins $I$ band, and several $V$-band images were obtained to measure the source color. RoboNet data were taken in SDSS-i band. From these follow-up observations, the second spike produced by the source star's caustic exit was captured with sufficient resolution to determine the angular source size. We note that the caustic exit was also covered by both OGLE and MOA surveys. See the upper middle panel of Figure 1.

The event continued after the caustic exit, and so did realtime modeling. Modeling conducted several days after the caustic exit revealed two important findings. First, it was predicted that there would be another pair of caustic crossings. Second, it was found that considering the microlens-parallax effect is important for the precise description of the observed light curve. With the progress of the event, the time of the next caustic crossing was refined. The microlensing community was informed of the predicted time of the caustic crossing, and follow-up observations were prepared accordingly. The third and fourth caustic crossings occurred successively at $\mathrm{HJD}^{\prime} \sim 6897$ and 6904, respectively. Although these crossings were missed by follow-up observations, they were partially captured by MOA survey observations. See the upper right panel in Figure 1. After these caustic crossings, the event gradually returned its baseline magnitude of $I \sim 18.75$.

Spitzer observations (Calchi Novati et al. 2015) were conducted during the period $6814<\mathrm{HJD}^{\prime}<6848$, which corresponded to the time when the event was in the trough region between the first two caustic crossings as seen from the ground. From the photometry of the Spitzer data, however, there exists no noticeable lensing signal, i.e., no variation of the source brightness. We discuss the reason for the absence of the Spitzer lensing signal in Section 3.

A very unusual characteristic of this event is that the light curve exhibits five peaks. The individual peaks occurred at $\mathrm{HJD}^{\prime} \sim 6698,6732,6854,6897$, and 6904, which are marked by the numbered arrows in Figure 1. Based on the shapes of the peaks, the first peak appears to be produced by the source's cusp approach, while the other four peaks were produced by caustic crossings. Multiple sets of caustic crossings can occur
Table 1

Error Bar Readjustment Factors

\begin{tabular}{lccr}
\hline \hline Data Set & $k$ & $\sigma_{\min }$ & $N_{\text {data }}$ \\
\hline OGLE & 1.808 & 0.002 & 3700 \\
MOA & 1.252 & 0.003 & 808 \\
$\mu$ FUN CTIO & 1.122 & 0.005 & 59 \\
RoboNet SSO (Dome A) & 0.692 & 0.025 & 61 \\
RoboNet SSO (Dome C) & 0.824 & 0.005 & 32 \\
RoboNet SAAO (Dome A) & 0.663 & 0.020 & 47 \\
RoboNet SAAO (Dome C) & 0.583 & 0.020 & 82 \\
\hline
\end{tabular}

when a source trajectory asymptotically passes a fold of a concave caustic. The existence of the multiple peaks in the light curve enables precise characterization of the lens system.

We conduct photometry of the data using the pipelines of the individual observation groups. The OGLE (Woźniak 2000; Udalski 2003) and MOA (Bond et al. 2001) pipelines are based on the Difference Image Analysis (DIA) technique (Alard \& Lupton 1998) and customized by the individual groups. The RoboNet and $\mu$ FUN data were reduced with the DANDIA pipeline (Bramich 2008) and the pySIS (Albrow et al. 2009), respectively. For the $\mu$ FUN CTIO data, photometry were additionally done with DoPHOT software (Schechter et al. 1993) in order for the source color measurement and color-magnitude diagram construction. We note that the quality of the MOA data at the baseline is not good, but their coverage of the caustic crossings is important in measuring $\theta_{\mathrm{E}}$. Therefore, we use MOA data taken when the source was magnified.

For the use of multiple data sets that are obtained with different telescopes and detectors and processed with different photometry softwares, it is required to readjust the error bars of the data sets. For this readjustment, we follow the standard procedure of Yee et al. (2012), where the error bars are renormalized by

$$
\sigma=k\left(\sigma_{0}^{2}+\sigma_{\min }^{2}\right)^{1 / 2},
$$

where $\sigma_{0}$ is the uncorrected error bar from the automated pipelines. We set the factor $\sigma_{\min }$ based on the scatter of data. The factor $k$ is set so that $\chi^{2}$ per degree of freedom (dof) becomes unity, i.e., $\chi^{2} /$ dof $=1$. We list the error bar readjustment factors in Table 1 along with the number of data points, $N_{\text {data }}$.

\section{Light Curve Modeling}

From the spike features, it is obvious that the event was produced by a lens composed of multiple components. Therefore, we start modeling of the observed light curve based on the binary-lens interpretation. For the simplest case where the relative lens-source motion is rectilinear, one needs seven principal parameters in order to describe the light curve of a binary-lens event. The first three parameters $\left(t_{0}, u_{0}, t_{\mathrm{E}}\right)$ are needed to describe the source approach to the lens, and they represent the time of the closest lens-source separation, the separation at that time, and the event timescale, respectively. Another three parameters $(s, q, \alpha)$ are used to describe the binary lens, and they denote the binary separation, mass ratio between the lens components, and the angle between the source trajectory and the line connecting the binary components, respectively. The caustic-crossing parts of a binary-lens event 
are affected by finite-source effects, and the last parameter $\rho$ is used to describe the deviation.

The light curve of the event exhibits caustic-crossing features, and thus we consider finite-source effects. We compute lensing magnifications affected by finite-source effects using the inverse ray-shooting technique. In computing finite-source magnifications, we take the surface brightness variation caused by limb-darkening into consideration. The surface brightness profile is approximated by a linear model, i.e.,

$$
\Sigma_{\lambda} \propto 1-\Gamma_{\lambda}\left(1-\frac{3}{2} \cos \phi\right),
$$

where $\lambda$ denotes the observed passband, $\Gamma_{\lambda}$ is the linear limbdarkening coefficient, and $\phi$ represents the angle between the line of sight and the normal to the surface of the source star. We determine the limb-darkening coefficients based on the source star's stellar type. It turns out that the source is an early K-type main-sequence star. See Section 4 for the detailed procedure of the source type determination. Based on the stellar type, we adopt the limb-darkening coefficients from the Claret (2000) catalog. The adopted $I$ - and $V$-band coefficients are $\Gamma_{I}=0.485$ and $\Gamma_{V}=0.676$, respectively. For the MOA $R$-band data, we use $\Gamma_{\mathrm{MOA}}=\left(\Gamma_{I}+\Gamma_{R}\right) / 2=0.535$, where $\Gamma_{R}=0.585$ is the $R$-band coefficient.

We search for the solution of the lensing parameters in two steps. In the first step, we divide the lensing parameters into two groups. We select $(s, q, \alpha)$ as grid parameters as lensing magnifications can vary dramatically with a small change in these parameters. We choose the other parameters, i.e., $\left(t_{0}, u_{0}, t_{\mathrm{E}}, \rho\right)$, as downhill parameters because lensing magnifications vary smoothly with the changes in the parameters. For the individual sets of the grid parameters, we then search for the set of the downhill parameters yielding the best $\chi^{2}$ using the Markov Chain Monte Carlo (MCMC) method. The total computation time for the grid search is $\sim 24 \mathrm{hr}$ using $176 \mathrm{CPUs}$. This initial search provides a $\chi^{2}$ map in the $s-q-\alpha$ parameter space, from which we identify local minima. We then refine each local minimum by allowing all of the parameters to vary. We note that the initial grid search is important for identifying degenerate solutions where different combinations of lensing parameters result in similar lensing light curves. For the case of OGLE-2014-BLG-0289, we identify a unique solution and find no solution with $\chi^{2}$ comparable to the best-fit solution.

In Figure 2, we present the model light curve (blue curve in the upper panel) of the solution obtained under the assumption of the rectilinear lens-source motion ("standard model"). The middle panel shows the residual from the model. For better visual comparison of the fit with data, we plot data points of only the OGLE, MOA, and $\mu$ FUN CTIO data sets. The binarylens parameters estimated by the model are $s \sim 1.6$ and $q \sim 0.9$. Although the standard model basically describes the overall light curve, it leaves considerable residuals. The major residuals occur near the first peak at $\mathrm{HJD}^{\prime} \sim 6700$ and the fifth peak at $\mathrm{HJD}^{\prime} \sim 6904$.

The inconsistency of the model with the data suggests the need to consider higher-order effects. Considering the long duration of the event, it is suspected that the assumption of a rectilinear lens-source motion may not be valid. There exist two major effects that can cause deviations of the relative lenssource motion from rectilinear. One such effect is caused by the orbital motion of the Earth around the Sun, i.e., the microlensparallax effect. The other is caused by the orbital motion of the binary lens itself, the lens-orbital effect. Therefore, we check whether the residuals from the standard model can be explained by these higher-order effects.

Incorporating higher-order effects requires including additional lensing parameters. In order to consider the microlensparallax effect, one needs two parameters: $\pi_{\mathrm{E}, N}$ and $\pi_{\mathrm{E}, E}$. They denote the north and east components $\pi_{\mathrm{E}}$ that represent the microlens-parallax vector projected onto the sky in the equatorial coordinate systems. The direction of $\pi_{\mathrm{E}}$ is the same as that of the relative lens-source motion (Gould 2000, 2004). Under the first-order approximation that the projected binary separation $s$ and the source trajectory angle $\alpha$ vary in constant rates, the lens-orbital effect is described by two parameters of $d s / d t$ and $d \alpha / d t$ (Albrow et al. 2000). With these parameters, we conduct additional modeling to check the improvement of the fit with the higher-order effects. In this modeling, we first separately consider the microlens-parallax ("parallax model") and lens-orbital effects ("orbit model"), and the simultaneously consider both of the effects ("parallax + orbit" model).

For events affected by microlens-parallax effects, there may exist a pair of degenerate solutions with $u_{0}>0$ and $u_{0}<0$ : "ecliptic degeneracy" (Skowron et al. 2011). This degeneracy arises because the source trajectories of the two degenerate solutions are in the mirror symmetry with respect to the binarylens axis. For the pair of the solutions subject to this degeneracy, the lensing parameters are approximately related by $\left(u_{0}, \alpha, \pi_{\mathrm{E}, N}, d \alpha / d t\right) \leftrightarrow-\left(u_{0}, \alpha, \pi_{\mathrm{E}, N}, d \alpha / d t\right)$. We check this degeneracy whenever microlens-parallax effects are considered in modeling.

We find that higher-order effects, particularly the microlensparallax effect, are important for explaining the residuals from the standard model. In Table 2, we present the $\chi^{2}$ values of the tested models. In Figure 3, we also present the cumulative distributions of $\chi^{2}$ as a function of time for the individual models. From the comparison of models, it is found that the fit improves by $\Delta \chi^{2} \sim 13103.1$ and 12938.5 by the microlensparallax and lens-orbital effects, respectively, with respect to the standard model. Among the two higher-order effects, it is found that the microlens-parallax effect is the main cause of the deviation from the standard model. The dominance of the microlens-parallax effect over the lens-orbital effect is found from the facts that (1) the "parallax" model yields substantially better fit than the "orbit" model (by $\Delta \chi^{2}=164.6$ ), (2) the parallax-only model can describe all five peaks, and (3) the further improvement from the parallax fit with the additional consideration of the lens-orbital effect $\left(\Delta \chi^{2}=17.3\right)$ is minor.

In Figure 2, we present the model light curve the orbit model (red curve in the upper panel) and the residual from the model (lower panel). It is found the model can describe the fifth peak, which could not be explained by the standard model, but it still cannot describe the first peak. To check the possibility that the assumption of the constant change rates of $d s / d t$ and $d \alpha / d t$ does not sufficiently describe lens-orbital effects, we conduct an additional modeling by fully considering the Keplerian orbital motion of the lens. This modeling requires two more parameters of $s_{\|}$and $d s_{\|} / d t$. These parameters represent binary separation (in units of $\theta_{\mathrm{E}}$ ) along the line of sight and the rate of separation change, respectively (Shin et al. 2011; Skowron et al. 2011). This modeling results in almost an identical $\chi^{2}$ to 


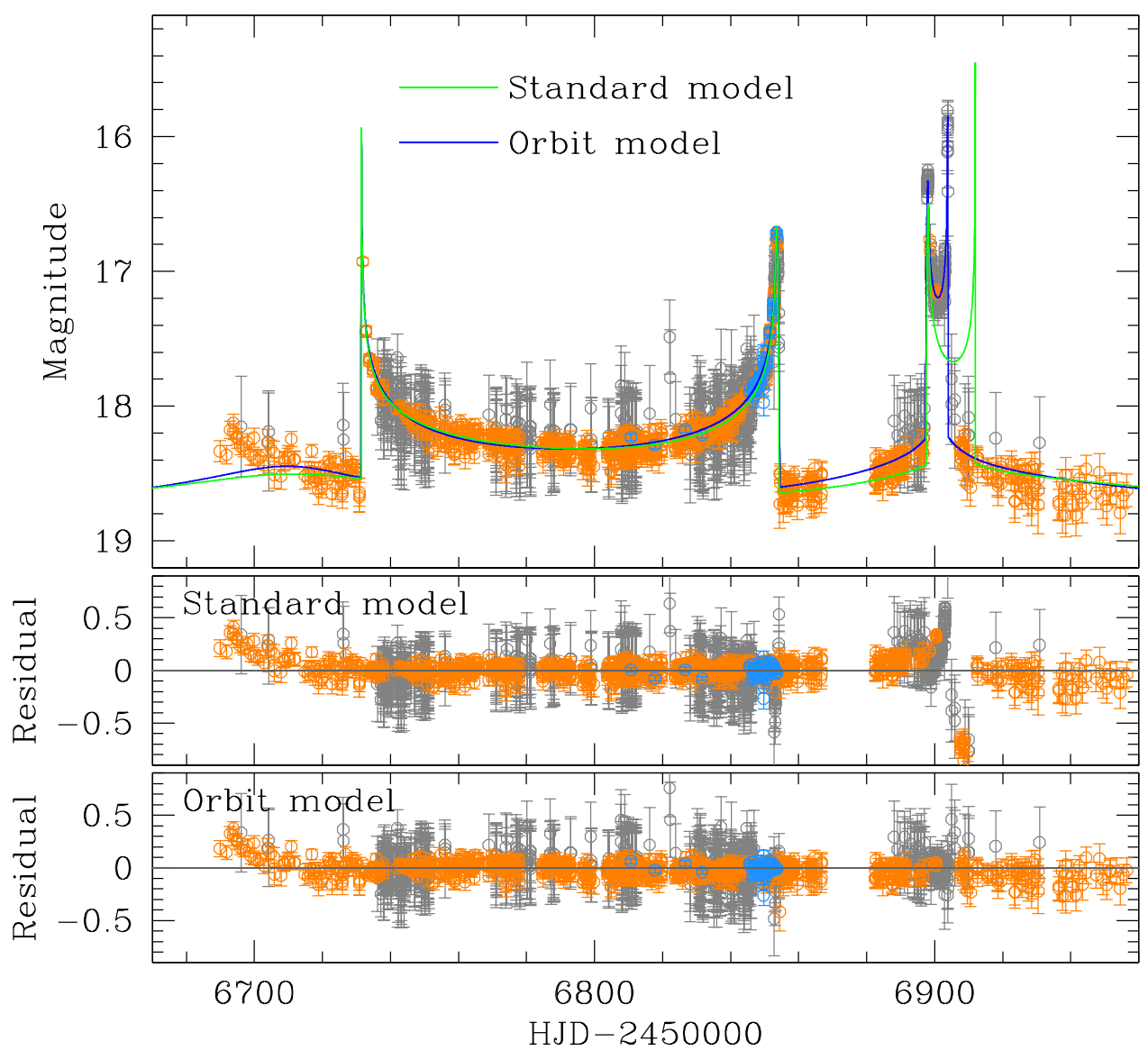

Figure 2. Model light curves of the "standard" (blue curve) and "orbit" (red curve) solutions. The middle and lower panels show the residuals from the individual models.

Table 2

Comparison of Models

\begin{tabular}{lr}
\hline \hline Model & \multicolumn{1}{c}{$\chi^{2}$} \\
\hline Standard & 17950.3 \\
Orbit & 5011.8 \\
Parallax $\left(u_{0}>0\right)$ & 4847.2 \\
Parallax $\left(u_{0}<0\right)$ & 4867.0 \\
Parallax + Orbit $\left(u_{0}>0\right)$ & 4829.9 \\
Parallax + Orbit $\left(u_{0}<0\right)$ & 4848.0 \\
\hline
\end{tabular}

that of the linear orbital motion solution. This confirms that the major cause of the deviation is the microlens-parallax effect.

In Table 3, we list the lensing parameters of the $u_{0}>0$ and $u_{0}<0$ solutions of the "parallax + orbit" model. Also presented are the fluxes of the source, $F_{s}$, and the blended light, $F_{b}$, that are measured based on the OGLE data. From the comparison of $u_{0}>0$ and $u_{0}<0$ solutions, it is found that the $u_{0}>0$ solution is slightly preferred over the $u_{0}<0$ solution by $\Delta \chi^{2}=18.1$. In Figure 4, we present the model light curve of the best-fit solution ("parallax+orbit" with $u_{0}>0$ ). To better show the fits around the caustic-crossing features, we also present the zoom of the regions in the upper panels. It is found that the model precisely describes all peaks.

Figure 5 shows the lens system geometry. In the geometry, we present the source trajectory (solid curve with an arrow) with respect to the caustic (cuspy closed curve) and the lens components (marked by $M_{1}$ and $M_{2}$ ) for the best-fit solution, i.e., $u_{0}>0$ solution of the parallax + orbit model. To show the variation of the lens positions and the resulting caustic due to lens-orbital effects, we present the caustics corresponding to four different times of the caustic crossings, although it is difficult to see the variation due to the minor lens-orbital effects. From the geometry, it is found that the first peak (at $\mathrm{HJD}^{\prime} \sim 6698$ ), which could be explained neither by the standard model nor by the orbital model, is explained by the source star's approach close to the cusp on the upper right part of the caustic. Being curved by the microlens-parallax effect, the source trajectory passes an outer edge of the lower left cusp and the model can describe the last peak, which could not be explained by the standard model.

We find that the absence of lensing signals in the Spitzer data is due to the fact that the space-based light curve during the Spitzer observation accidentally corresponds to a region where the light curve is very flat. In the middle panel of Figure 4, we present the light curve expected to be observed in space using the Spitzer telescope (blue curve). We note that the Spitzer light curve is constructed based on the microlens-parallax parameters determined from the ground-based data. The region represented by a left-right arrow and marked by "Spitzer observation" denotes the period during which Spitzer observations were conducted. It shows that this region of the light curve is very flat, and thus there is no noticeable lensing signal in the Spitzer data. In the lower panel of Figure 5, we present the source trajectory (dotted curve with an arrow) that is expected to be seen from the Spitzer telescope. The consistency of the predicted model with the flat Spitzer data further supports 


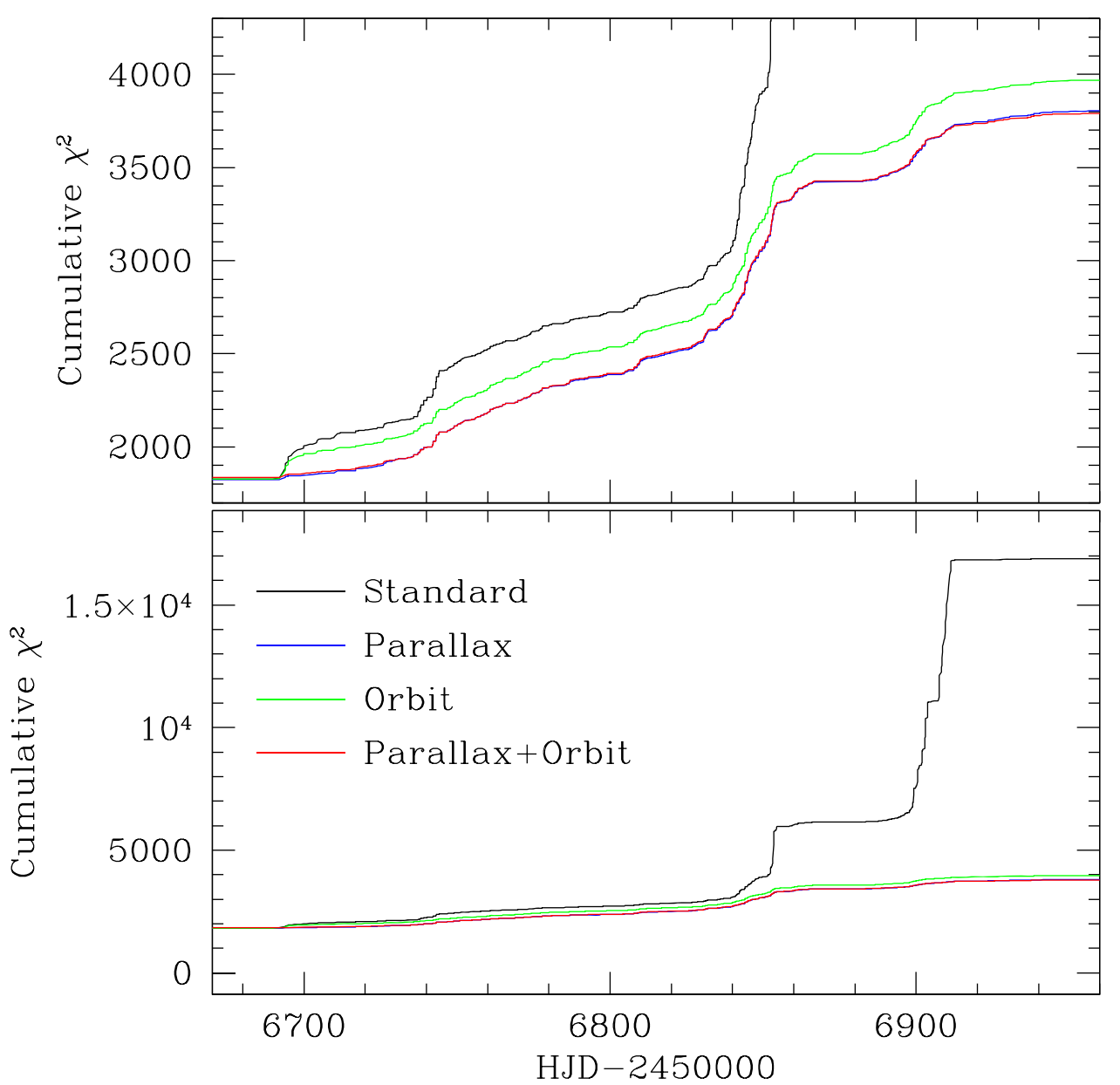

Figure 3. Cumulative distributions of $\chi^{2}$ as a function of time for the tested models. To better show the differences between models considering higher-order effects, we present the zoom of the distributions in the upper panel. We note that the $\chi^{2}$ difference between the "parallax" and "parallax+orbit" models is so small that it is difficult to distinguish the two distributions within the line width.

Table 3

Best-fit Lensing Parameters

\begin{tabular}{lcc}
\hline \hline \multirow{2}{*}{ Parameter } & \multicolumn{2}{c}{ Value } \\
\cline { 2 - 3 } & $u_{0}>0$ & $u_{0}<0$ \\
\hline$\chi^{2}$ & 4829.9 & 4848.8 \\
$t_{0}\left(\mathrm{HJD}^{\prime}\right)$ & $6820.781 \pm 0.327$ & $6802.729 \pm 0.391$ \\
$u_{0}$ & $0.049 \pm 0.001$ & $-0.031 \pm 0.001$ \\
$t_{\mathrm{E}}($ days $)$ & $144.43 \pm 0.24$ & $162.59 \pm 1.40$ \\
$s$ & $1.64 \pm 0.01$ & $1.59 \pm 0.01$ \\
$q$ & $0.81 \pm 0.01$ & $1.06 \pm 0.01$ \\
$\alpha(\mathrm{rad})$ & $2.852 \pm 0.004$ & $-2.739 \pm 0.004$ \\
$\rho\left(10^{-3}\right)$ & $0.52 \pm 0.01$ & $0.57 \pm 0.01$ \\
$\pi_{\mathrm{E}, N}$ & $0.111 \pm 0.002$ & $-0.071 \pm 0.005$ \\
$\pi_{\mathrm{E}, E}$ & $-0.104 \pm 0.002$ & $-0.051 \pm 0.006$ \\
$d s / d t\left(\mathrm{yr}^{-1}\right)$ & $-0.04 \pm 0.02$ & $-0.01 \pm 0.01$ \\
$d \alpha / d t\left(\mathrm{yr}^{-1}\right)$ & $0.10 \pm 0.01$ & $-0.07 \pm 0.01$ \\
$\left(F_{s} / F_{b}\right)_{\mathrm{OGLE}}$ & $0.097 / 0.392$ & $0.108 / 0.380$ \\
\hline
\end{tabular}

the correctness of the solution determined from the groundbased data.

If the source is a binary, the orbital motion of the source can also induce long-term deviations in lensing light curves: "xallarap effect" (Poindexter et al. 2005; Rahvar \& Dominik 2009). We, therefore, check the xallarap possibility of the deviation. Considering xallarap effects requires five parameters in addition to the principal parameters. These include the north and east components of the xallarap vector, $\xi_{\mathrm{E}, N}$ and $\xi_{\mathrm{E}, E}$, the orbital period, the phase angle, and the inclination of the orbit. See the appendix of Han et al. (2016a) for details about the xallarap parameters. We find a best-fit xallarap model with an orbital period $P \sim 0.5$ year, but the model is worse than the best-fit parallax+orbit model by $\Delta \chi^{2}=118.3$, which is significant enough to exclude the xallarap interpretation.

\section{Characterizing the Lens \\ 4.1. Physical Lens Parameters}

To uniquely determine the lens mass, the angular Einstein radius, in addition to the microlens parallax, needs to be estimated. The angular Einstein radius is determined by

$$
\theta_{\mathrm{E}}=\frac{\theta_{*}}{\rho} .
$$

We measure the normalized source radius $\rho$ from the analysis of the caustic-crossing parts of the light curve. We note that the third peak was resolved with a sufficient coverage for the $\rho$ 

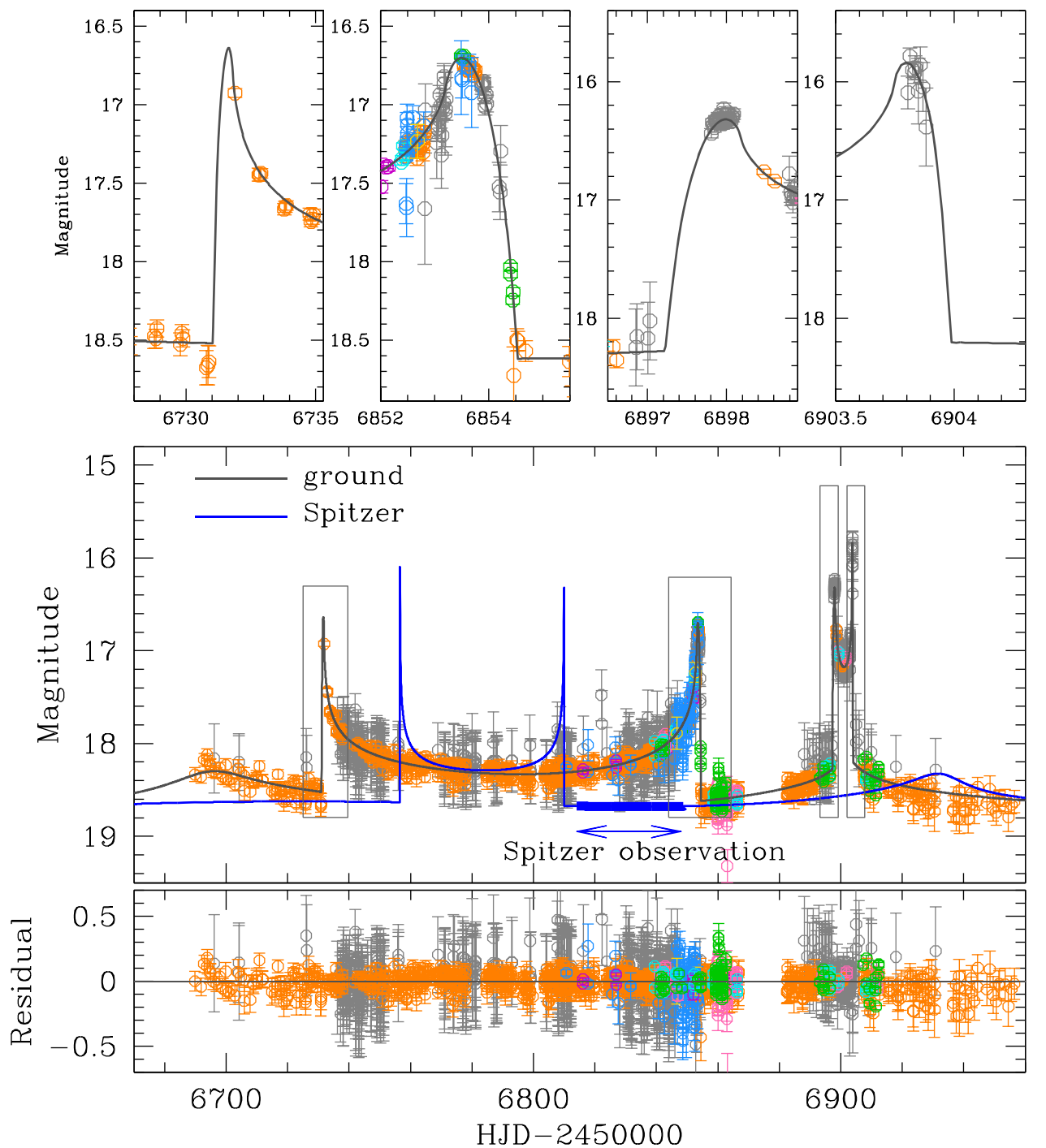

Figure 4. Best-fit model light curve (black solid curve). Upper panels show the model fits around the regions enclosed by boxes in the middle panel. The lower panel shows the residual from the model. The blue curve in the middle panel represents the light curve expected to be observed in space using the Spitzer telescope. The region represented by a left-right arrow and marked "Spitzer observation" denotes the period during which Spitzer observations were conducted $(2456814<$ HJD $<2456848)$.

measurement. See the upper panels of Figure 4. To determine $\theta_{\mathrm{E}}$, then, one needs to estimate the angular source radius $\theta_{*}$.

We determine the angular source radius based on the dereddened color $(V-I)_{0}$ and brightness $I_{0}$ of the source star. For the color and brightness determinations, we use the method of Yoo et al. (2004). In this method, $(V-I)_{0}$ and $I_{0}$ are determined from the offsets in color $\Delta(V-I)$ and brightness $\Delta I$ with respect to the centroid of the red giant clump (RGC), for which the intrinsic color and brightness are known. In Figure 6, we present the color-magnitude diagram of stars in the neighboring region around the source star. The colormagnitude diagram is constructed based on the DoPHOT photometry of the $\mu$ FUN CTIO data. It is aligned to the OGLEIII photometric system by shifting the clump magnitude according to the extinction, $A_{I}=1.77$, and the reddening, $E(V-I)=1.46$, toward the field based on the OGLE-III extinction map (Nataf et al. 2013). We mark the positions of the RGC centroid and the source by a red and blue dots. From the offsets in color $\Delta(V-I)=-0.39$ and magnitude $\Delta I=4.70$ and the known dereddened values of the RGC,
$(V-I, I)_{\mathrm{RGC}, 0}=(1.06,14.41)$ (Bensby et al. 2013; Nataf et al. 2013), we find that the dereddeded color and brightness of the source star are $(V-I, I)_{0}=(V-I, I)_{\mathrm{RGC}}+[\Delta(V-I)$, $\Delta I]=(0.93 \pm 0.05,19.11 \pm 0.01)$, indicating that the source is an early $\mathrm{K}$-type main-sequence star. We then convert $(V-I)_{0}$ into $(V-K)_{0}$ using the $V-I / V-K$ relation of Bessell \& Brett (1988) and employ the color/surface brightness relation of Kervella et al. (2004) to find $\theta_{*}=0.61 \pm 0.04 \mu$ as. We estimate that the angular Einstein radius is

$$
\theta_{\mathrm{E}}=1.17 \pm 0.09 \text { mas. }
$$

Here we adopt the source distance that is estimated using the relation $D_{\mathrm{S}}=D_{\mathrm{GC}} /(\cos l+\sin l / \tan \phi)$ (Nataf et al. 2013), where $D_{\mathrm{GC}}=8160 \mathrm{pc}$ is the galactocentric distance, $l$ is the galactic longitude, and $\phi \sim 40^{\circ}$ is the angle between the semimajor axis of the bulge and the line of sight. With $l=0.9$, the adopted source distance is $D_{\mathrm{S}}=8011 \mathrm{pc}$. In combination of the event timescale, the measured angular Einstein radius 

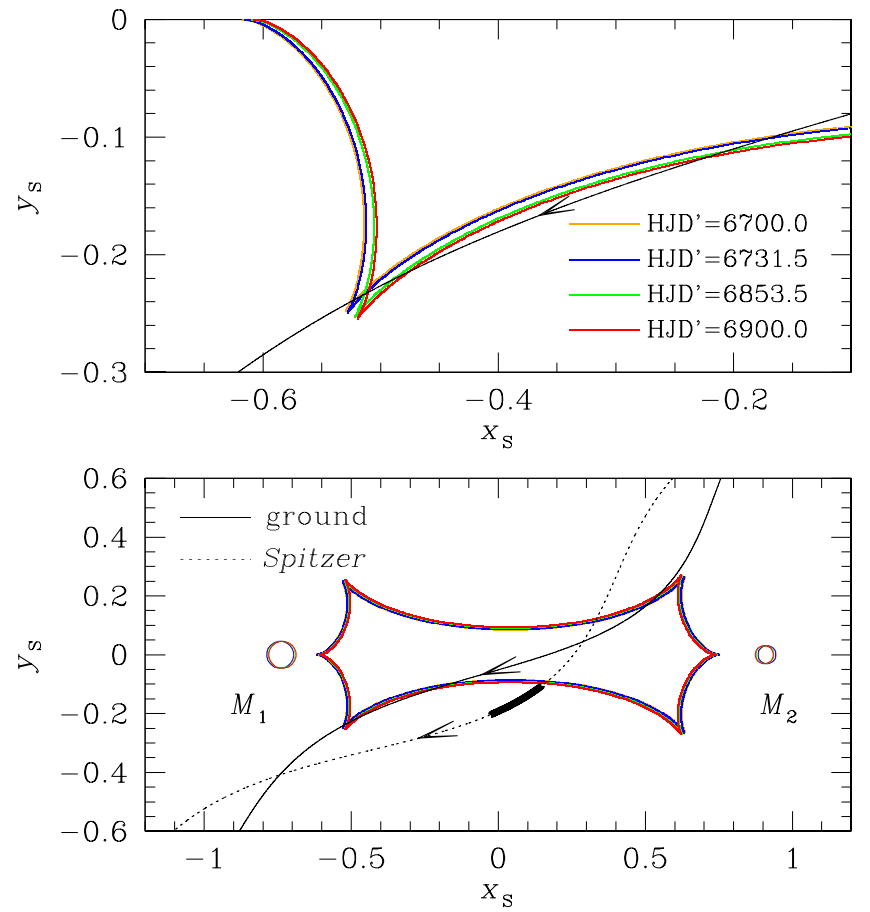

Figure 5. Lens system geometry showing the source trajectory (solid curve with an arrow) with respect to the caustic (cuspy closed curve) and the lens components (marked by $M_{1}$ and $M_{2}$ ). The upper panel shows the enlargement of the lower left region of the caustic. The caustics at four different times are presented in different colors. The dotted curve represents the source trajectory seen in space from the Spitzer telescope. The thick line on the Spitzer source trajectory represents the time during which the event was observed by the Spitzer telescope.

yields the relative lens-source proper motion of

$$
\mu=\frac{\theta_{\mathrm{E}}}{t_{\mathrm{E}}}=2.97 \pm 0.21{\mathrm{mas} \mathrm{yr}^{-1} .} .
$$

With both measured $\pi_{\mathrm{E}}$ and $\theta_{\mathrm{E}}$, the total mass $M=M_{1}+M_{2}$ is determined using the relation in Equation (2) and the masses of the individual components are determined by

$$
M_{1}=\frac{M}{1+q} ; \quad M_{2}=\frac{q M}{1+q} .
$$

The distance to the lens is determined by the relation

$$
D_{\mathrm{L}}=\frac{\mathrm{au}}{\pi_{\mathrm{E}} \theta_{\mathrm{E}}+\pi_{\mathrm{S}}},
$$

where $\pi_{\mathrm{S}}=\mathrm{au} / D_{\mathrm{S}}$ represents the parallax of the source star. The projected separation between the lens components is determined by $a_{\perp}=s D_{\mathrm{L}} \theta_{\mathrm{E}}$.

In Table 4, we list the physical parameters of the lens. We find that the lens is a binary composed of two M dwarfs with masses

$$
M_{1}=0.52 \pm 0.04 M_{\odot}
$$

and

$$
M_{2}=0.42 \pm 0.03 M_{\odot} .
$$

The estimated distance to the lens is

$$
D_{\mathrm{L}}=3.3 \pm 0.3 \mathrm{kpc} \text {. }
$$

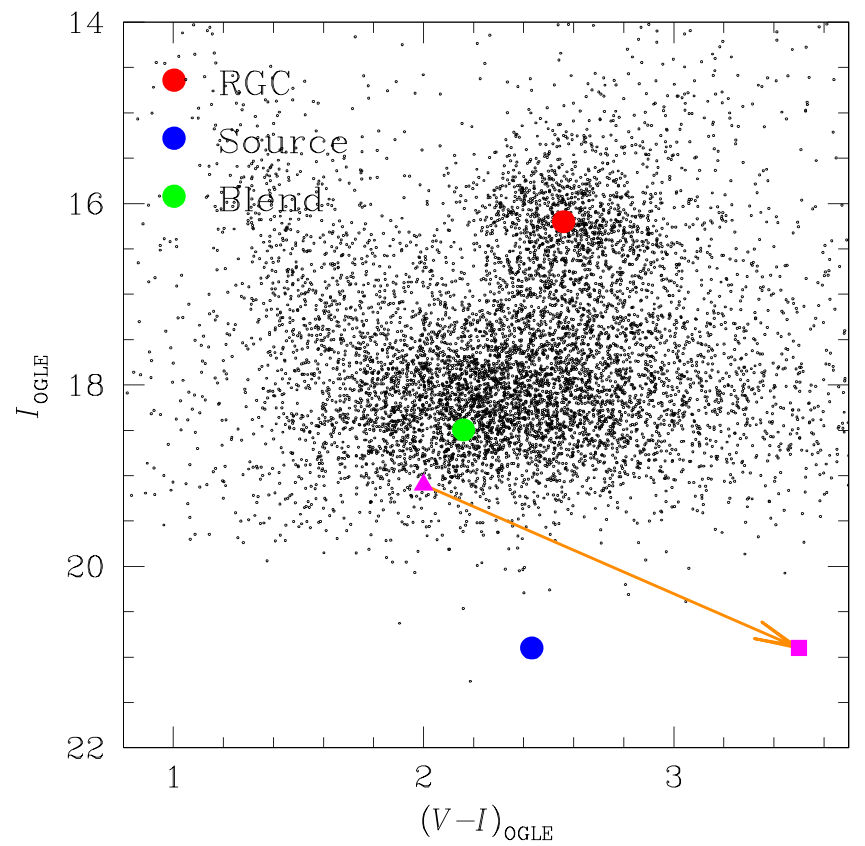

Figure 6. Source location with respect to the centroid of the red giant clump (RGC) in the color-magnitude diagram. Also marked are the positions of the blend. The filled triangle and square dots denote the lens positions under the assumptions of no and full extinction, respectively.

Table 4

Physical Lens Parameters

\begin{tabular}{lc}
\hline \hline Parameter & Value \\
\hline Primary mass & $0.52 \pm 0.04 M_{\odot}$ \\
Companion mass & $0.42 \pm 0.03 M_{\odot}$ \\
Projected separation & $6.4 \pm 0.5 \mathrm{au}$ \\
Distance to the lens & $3.3 \pm 0.3 \mathrm{kpc}$ \\
$(\mathrm{KE} / \mathrm{PE})_{\perp}$ & 0.03 \\
\hline
\end{tabular}

The projected separation between the lens components is

$$
a_{\perp}=6.4 \pm 0.5 \mathrm{au} .
$$

Also presented in Table 4 is the ratio of the transverse kineticto-potential energy ratio $(\mathrm{KE} / \mathrm{PE})_{\perp}$. The ratio is computed from the measured lensing parameters by

$$
\left(\frac{\mathrm{KE}}{\mathrm{PE}}\right)_{\perp}=\frac{\left(a_{\perp} / \mathrm{au}\right)^{3}}{8 \pi^{2}\left(M / M_{\odot}\right)}\left[\left(\frac{1}{s} \frac{d s}{d t}\right)^{2}+\left(\frac{d \alpha}{d t}\right)^{2}\right] .
$$

The ratio should be less than unity to be a bound system, i.e., $(\mathrm{KE} / \mathrm{PE})_{\perp} \leqslant \mathrm{KE} / \mathrm{PE}<1.0$. It is found that the determined value $(\mathrm{KE} / \mathrm{PE})_{\perp}=0.03$ meets this requirement. Due to the small lens-orbital effect, the ratio is small, probably due to the alignment of the lens components along the line of sight.

\subsection{Is the Blend the Lens?}

In Figure 6, we mark the location of the blend in the colormagnitude diagram. Then, a question is whether the blend is the lens itself. The intrinsic color corresponding to the mass of the primary lens, $\sim 0.5 M_{\odot}$, is $(V-I)_{\mathrm{L} 1,0} \sim 1.9$. Were it not for any extinction and reddening, the apparent color and brightness of the primary lens would be 

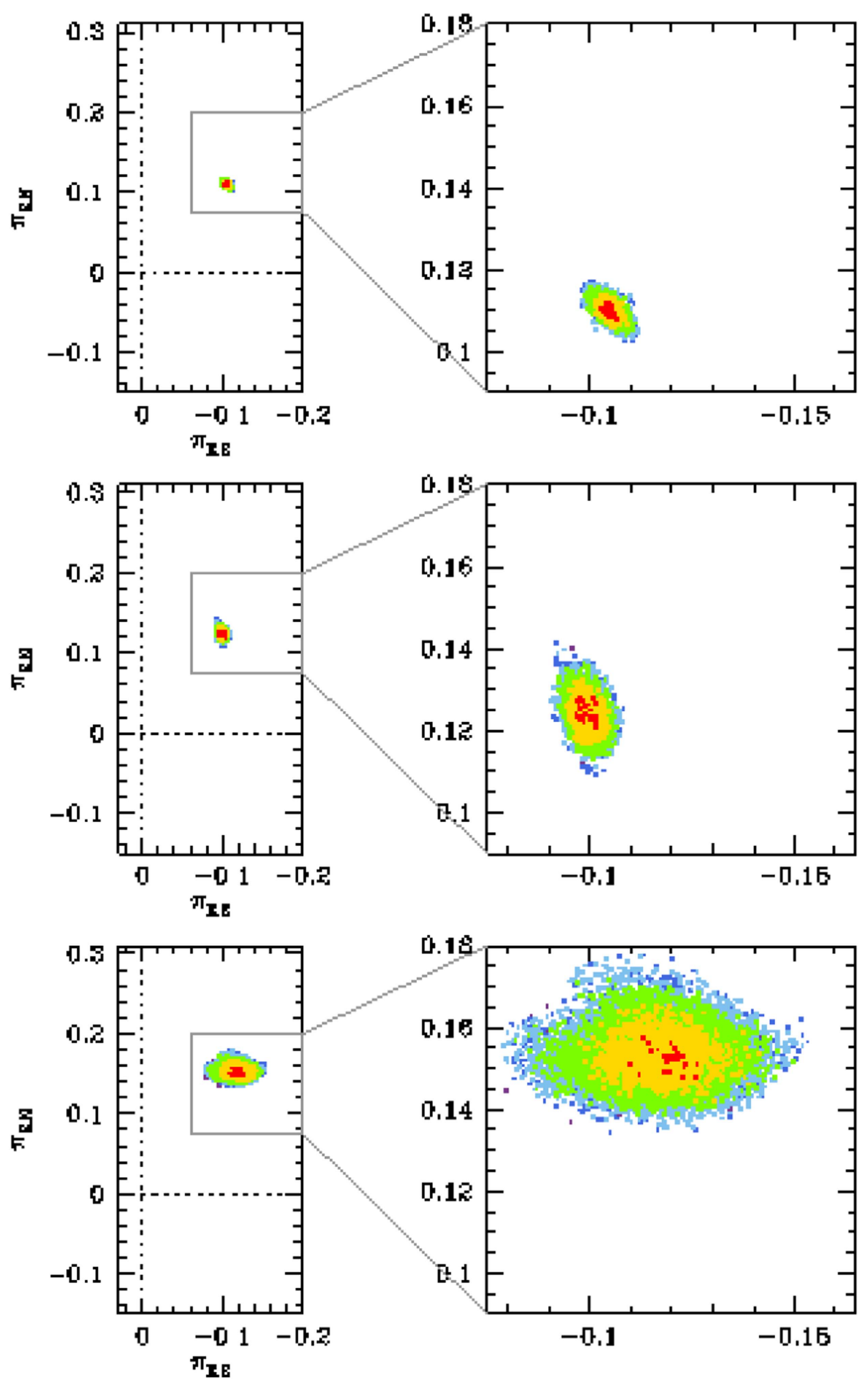

Figure 7. $\Delta \chi^{2}$ distributions of MCMC chains obtained from modeling runs based on different data sets. The distribution in the upper panel is obtained based on all data. The distribution in the middle panel is based on the data where data points in the region $6890<\mathrm{HJD}^{\prime}<6910$ are excluded. The distribution in the lower panel is based on the data, with additionally excluded data points in the region $6850<\mathrm{HJD}^{\prime}<6860$. Dots marked in different colors represent chains with $\Delta \chi^{2}<1$ (red), 4 (yellow), 9 (green), 16 cyan (magenta), and 25 (blue). Right panels show the zoom of the regions enclosed by a box in the corresponding left panels.

$(V-I)_{\mathrm{L} 1} \sim(V-I)_{\mathrm{L} 1,0} \sim 1.9$ and $I_{\mathrm{L} 1}=M_{I, 1}+5 \log D_{\mathrm{L}}-$ $5 \sim 19.6$. Here $M_{I, 1} \sim 7.0$ represents the absolute magnitude corresponding to the mass of the primary lens. Considering that the primary is accompanied by a slightly less-massive companion, the combined color and brightness of the lens would be $(V-I, L)_{\mathrm{L}} \sim(2.0,19.1)$. If the blend is in the bulge, on the other hand, it would have experienced the same amount of extinction $A_{I} \sim 1.8$ and reddening $E(V-I) \sim 1.5$ as those of 
the source star. We mark the positions of the lens in the colormagnitude diagram under the assumptions of no and full extinction by a filled triangle and a square points, respectively. Considering the distance to the lens of $D_{\mathrm{L}} \sim 3.3 \mathrm{kpc}$, it is likely that the lens is inside the obscuring dust. In this case, the lens on the color-magnitude diagram will be located on the line connecting the two points. It is found that the blend is away from this line, suggesting that the blend is not the lens. This line of reasoning is supported by the astrometric offset $\Delta \theta \sim 0$ !' 14 between the source position (measured from the difference image near the peak of the event) and the OGLE catalog position (which is dominated by the blend because it is $\sim 2$ mag brighter than the source).

\section{Discussion}

The event OGLE-2014-BLG-0289 is very unusual in the sense that its light curve exhibits five peaks, of which two were partially covered and the others were densely resolved. In this section, we demonstrate that the quintuple peaks help to determine the microlens parallax with improved accuracy and precision.

For this demonstration, we conduct additional modeling runs with data sets where parts of the data points are excluded. In the first run, we exclude data points in the region $6890<\mathrm{HJD}^{\prime}<6910$ to simulate the case where the fourth and fifth peaks were missed ("case 1"). In the second run, we additionally exclude data points in the region $6850<\mathrm{HJD}^{\prime}<6860$ to simulate the case where the third peak was additionally missed ("case 2 ").

In Figure 7 , we present the $\Delta \chi^{2}$ distributions of MCMC chains in the $\pi_{\mathrm{E}, E}-\pi_{\mathrm{E}, N}$ parameter space obtained from the modeling runs with three different data sets. Dots marked in different colors represent chains with $\Delta \chi^{2}<1$ (red), 4 (yellow), 9 (green), 16 cyan (magenta), and 25 (blue). The upper panel is based on all data and the distributions in the middle and lower panels are for "case 1" and "case 2," respectively. The right panels show the enlarged view of the regions enclosed by a box in the corresponding left panels.

By comparing the distributions, we find that the microlensparallax parameters determined based on the partial data sets differ from those based on the full data set by $\Delta\left(\pi_{\mathrm{E}, N}, \pi_{\mathrm{E}, E}\right) \sim$ $(0.01,0.01)$ for the "case 1 " and $\sim(0.04,0.02)$ for the "case 2." This indicates that the coverage of the peaks affects the accuracy of the $\pi_{\mathrm{E}}$ determination. Furthermore, the uncertainties of the determined microlens-parallax parameters increase as fewer caustics are resolved, suggesting that the peak coverage also affects the precision of the $\pi_{\mathrm{E}}$ determination. These results demonstrate that the resolution of the individual peaks provides important constraints on the determinations of the lens parameters. We note that there was a discovery of an additional lensing event with quintuple peaks. The event, Gaial6aye, showed a complex light curve with four caustic crossings and a fifth brightening likely due to a cusp approach (Mróz et al. 2016; Wyrzykowski et al. 2017).

\section{Conclusion}

We analyzed the binary-microlensing event OGLE-2014BLG-0289. The light curve of the event exhibited very unusual five peaks, where four peaks were produced by caustic crossings and the other peak by a cusp approach. We found that the quintuple-peak features of the light curve enabled us to precisely and accurately measure the microlensing parallax $\pi_{\mathrm{E}}$. The three resolved caustics allowed us to precisely measure the angular Einstein radius $\theta_{\mathrm{E}}$. From the combination of $\pi_{\mathrm{E}}$ and $\theta_{\mathrm{E}}$, the physical parameters of the lens were uniquely determined. We found that the lens was a binary composed of two $\mathrm{M}$ dwarfs with masses $M_{1}=0.49 \pm 0.04 M_{\odot}$ and $M_{2}=0.39 \pm 0.03 M_{\odot}$ separated in projection by $a_{\perp}=6.2 \pm 0.5 \mathrm{au}$. The lens was located in the disk with a distance of $D_{\mathrm{L}}=3.4 \pm 0.3 \mathrm{kpc}$.

Work by C. Han was supported by the grant (2017R1A4A1015178) of National Research Foundation of Korea. The OGLE project has received funding from the National Science Centre, Poland, grant MAESTRO 2014/14/A/ST9/ 00121 to A. Udalski. OGLE Team thanks Profs. M. Kubiak and G. Pietrzyński for their contribution to the OGLE photometric data set presented in this paper. The MOA project is supported by JSPS KAKENHI grant Nos. JSPS24253004, JSPS26247023, JSPS23340064, JSPS15H00781, and JP16H06287. Work by A. Gould was supported by JPL grant 1500811 . Work by J. C. Yee was performed in part under contract with the California Institute of Technology (Caltech)/Jet Propulsion Laboratory (JPL) funded by NASA through the Sagan Fellowship Program executed by the NASA Exoplanet Science Institute. We acknowledge the high-speed internet service (KREONET) provided by Korea Institute of Science and Technology Information (KISTI).

Software: OGLE pipeline (Woźniak 2000; Udalski 2003), MOA pipeline (Bond et al. 2001), the DANDIA pipeline (Bramich 2008), pySIS (Albrow et al. 2009), DoPHOT (Schechter et al. 1993).

\section{ORCID iDs}

C. Han (ib https://orcid.org/0000-0002-2641-9964

V. Bozza (iD https://orcid.org/0000-0003-4590-0136

J. Skowron (1) https://orcid.org/0000-0002-2335-1730

P. Pietrukowicz (D) https://orcid.org/0000-0002-2339-5899

D. P. Bennett (i) https://orcid.org/0000-0001-8043-8413

A. Fukui (iD https://orcid.org/0000-0002-4909-5763

Y. Itow (iD https://orcid.org/0000-0002-8198-1968

K. Kawasaki iD https://orcid.org/0000-0003-2006-1735

N. Koshimoto (iD https://orcid.org/0000-0003-2302-9562

S. Miyazaki ib https://orcid.org/0000-0002-1962-904X

D. Suzuki (i) https://orcid.org/0000-0002-5843-9433

E. Bachelet (i) https://orcid.org/0000-0002-6578-5078

N. Kains (i) https://orcid.org/0000-0001-8803-6769

C. Snodgrass (i) https://orcid.org/0000-0001-9328-2905

I. A. Steele (iD https://orcid.org/0000-0001-8397-5759

J. Wambsganss (i) https://orcid.org/0000-0002-8365-7619

R. W. Pogge (iD https://orcid.org/0000-0003-1435-3053

\section{References}

Alard, C., \& Lupton, R. H. 1998, ApJ, 503, 325

Albrow, M. D., Beaulieu, J.-P., Caldwell, J. A. R., et al. 2000, ApJ, 534, 894 Albrow, M. D., Horne, K., Bramich, D. M., et al. 2009, MNRAS, 397, 2099 Alcock, C., Allsman, R. A., Axelrod, T. S., et al. 1995, ApJ, 445, 133

An, J. H., \& Gould, A. 2001, ApJL, 563, 111

Aubourg, E., Bareyre, P., Brehin, S., et al. 1995, A\&A, 301, 1

Batista, V., Gould, A., Dieters, S., et al. 2011, A\&A, 529, 102

Bensby, T., Yee, J. C., Feltzing, S., et al. 2013, A\&A, 549, A147 Bessell, M. S., \& Brett, J. M. 1988, PASP, 100, 1134

Bond, I. A., Abe, F., Dodd, R. J., et al. 2001, MNRAS, 327, 868 Bramich, D. M. 2008, MNRAS, 386, L77

Calchi Novati, S., Gould, A., Udalski, A., et al. 2015, ApJ, 804, 20

Choi, J.-Y., Shin, I.-G., Park, S.-Y., et al. 2012, ApJ, 751, 41

Claret, A. 2000, A\&A, 363, 1081

Gould, A. 1992, ApJ, 392, 442 
Gould, A. 1994, ApJL, 421, 75

Gould, A. 2000, ApJ, 542, 785

Gould, A. 2004, ApJ, 606, 319

Han, C., Jung, Y. K., Udalski, A., et al. 2016a, ApJ, 822, 75

Han, C., Udalski, A., Lee, C.-U., et al. 2016b, ApJ, 827, 11

Kervella, P., Bersier, D., Mourard, D., et al. 2004, A\&A, 28, 587

Mróz, P., Wyrzykowski, L., Rybicki, K., et al. 2016, ATel, 9770, 1

Nataf, D. M., Gould, A., Fouqué, P., et al. 2013, ApJ, 769, 88

Nemiroff, R. J., \& Wickramasinghe, W. A. D. T. 1994, ApJL, 424, 21

Poindexter, S., Afonso, C., Bennett, D. P., et al. 2005, ApJ, 633, 914

Pratt, M. R., Alcock, C., Allsman, R. A., et al. 1996, in Proc. IAU Symp. 173 , Astrophysical Applications of Gravitational Lensing, ed. C. S. Kochanek \& J. N. Hewitt (Dordrecht: Kluwer), 221

Rahvar, S., \& Dominik, M. 2009, MNRAS, 392, 1193
Refsdal, S. 1966, MNRAS, 134, 315

Schechter, P. L., Mateo, M., \& Saha, A. 1993, PASP, 105, 1342

Shin, I.-G., Udalski, A., Han, C., et al. 2011, ApJ, 735, 85

Skowron, J., Udalski, A., Gould, A., et al. 2011, ApJ, 738, 87

Street, R. A., Udalski, A., Calchi Novati, S., et al. 2016, ApJ, 819, 93

Sumi, T., Abe, F., Bond, I. A., et al. 2003, ApJ, 591, 204

Udalski, A. 2003, AcA, 53, 291

Udalski, A., Szymański, M., Kalużny, J., et al. 1994, AcA, 44, 1

Udalski, A., Yee, J. C., Gould, A., et al. 2015, ApJ, 799, 237

Woźniak, P. R. 2000, AcA, 50, 421

Wyrzykowski, L., Mróz, P., Rybicki, K., et al. 2017, ATel, 10341, 1

Yee, J. C., Shvartzvald, Y., Gal-Yam, A., et al. 2012, ApJ, 755, 102

Yoo, J., DePoy, D. L., Gal-Yam, A., et al. 2004, ApJ, 603, 139

Zhu, W., Calchi Novati, S., Gould, A., et al. 2016, ApJ, 825, 60 